\title{
CONTRIBUTO PARA UM CICS NACIONAL: TABELAS DE PRODUTOS, DE PROPRIEDADES, DE FORMAS DE INFORMAÇÃO, DE GESTÃO E DIREÇÃO DE PROJETO E DE ELEMENTOS
}

\author{
Paula Couto $^{(1)}$, Maria João Falcão Silva ${ }^{(1)}$, Fernando Pinho ${ }^{(2)}$ \\ (1) Laboratório Nacional de Engenharia Civil (LNEC), Lisboa \\ (2) CERIS, FCT-UNL, Monte da Caparica
}

\begin{abstract}
Resumo
O aumento da complexidade no setor de Arquitetura, Engenharia, Construção e Operação (AECO) e o desenvolvimento da metodologia Building Information Modelling (BIM) vieram gerar mais informação relacionada com os empreendimentos de construção, criando a necessidade de interoperabilidade entre sistemas e promovendo a ligação entre a normalização dos objetos BIM e os Sistemas de Classificação de Informação da Construção (Construction Information Classification Systems - CICS). Os CICS permitem a organização de forma racional, o apoio e a recuperação de informação, tendo como objetivo a troca de informação ao longo de todo o ciclo de vida dos ativos construídos.

$\mathrm{O}$ estudo apresentado no presente artigo pretende-se efetuar uma proposta para o desenvolvimento das tabelas de classificação dos "Produtos", das "Propriedades", das "Formas de Informação" e "Gestão e Direção de Projeto" para o CICS nacional, assentes na estrutura padrão proposta pela ISO 12006-2 e na adoção do sistema de classificação Uniclass 2015. O desenvolvimento das tabelas dos "Produtos" e das "Propriedades" compreende a análise da normalização e da marcação CE de produtos da construção e das características e/ou propriedades dos mesmos, estas últimas para o caso de estudo dos agregados, através da informação presente nas normas, no Anexo informativo ZA, e nos objetos das tabelas do CICS nacional. As tabelas "Formas de Informação" e "Gestão e Direção de Projeto" compreendem, respetivamente, uma interligação entre elementos integrantes de um projeto e a adaptação, ao contexto nacional, dos documentos utilizados em toda a logística e gestão de qualquer empreendimento. As tabelas propostas são sustentadas por variadas fontes internacionais e nacionais, resultando numa codificação para objetos BIM.
\end{abstract}

\section{Introdução}

A evolução na sociedade atual permitiu que cada vez mais haja uma quantidade maior de informação trocada entre os mais diversos setores, sendo o setor da construção um exemplo desta situação. Existe então uma tendência crescente da quantidade de informação produzida e 
das exigências da qualidade da mesma. Para tal a ISO tem produzido Normas com o objetivo de uniformizar a classificação e troca de informação, como é o caso da ISO 12006-2:2015 [1] e ISO 12006-3:2007 [2] que têm como objetivo orientar a estruturação de sistemas de classificação da informação na construção. Num âmbito geral, o sistema de classificação definido de acordo com estas Normas terá que abranger todo o ciclo de vida do empreendimento e conseguir tratar uma grande quantidade de informação proveniente de uma grande variedade de trabalhos de construção e serviços adjacentes [3].

A organização do conjunto de informação relativa ao ciclo de vida de um projeto é um passo da maior importância no que respeita à interligação de todos os intervenientes ao longo do ciclo de vida de um empreendimento de construção. A linguagem classificada segundo os mesmos princípios é a chave do sucesso para uma gestão, coordenação e interação em obra eficientes, dando contributo para uma melhoria muito significativa dos custos e prazos das empreitadas. Apesar de, em Portugal, ainda não existir nem uma regulamentação governamental definida e obrigatória para a utilização do BIM, nem um Sistemas de Classificação de Informação da Construção (CICS) nacional, devido à sua importância e à necessidade de implementação na área da Engenharia Civil, a International Organization for Standardization (ISO), através do Comité Técnico 59 (TC59), tem tido essa preocupação e como principal finalidade a criação e desenvolvimento de normas, com vista à uniformização de toda a informação no setor [4].

Na definição das características ou das informações relevantes que subsistem no modelo BIM, para que este não venha a conter excesso de informação, desenvolveram-se especificações de níveis de definição. No contexto nacional, para a definição das características relevantes, desenvolveram-se níveis de definição com base no contexto internacional, determinado pelo Comité Europeu de Normalização (CEN/TC 442), que promove e acompanha os desenvolvimentos da normalização BIM. Este define os Levels of Definitions (LOD), e mais recentemente os Levels of Information Needed [5-6], permitindo estabelecer conceitos e princípios para esclarecer os níveis e necessidades de informação durante o ciclo de vida do projeto e/ou empreendimento, de acordo com a metodologia BIM. Os LOD definem o quão detalhada deve ser a informação, o comportamento e a apresentação dos objetos, geométrica e/ou alfanumericamente, na metodologia BIM, para permitir consistência, eficiência e interoperabilidade em toda a indústria da construção. Os LOD estão divididos em dois grupos, nomeadamente, o LOG (Level of Geometry) e o LOI (Level of Information). O LOG define o detalhe da informação geométrica e o LOI define o detalhe das informações alfanuméricas que estão diretamente relacionadas com a geometria [7].

Neste sentido, a necessidade de integrar no CICS nacional os produtos da construção e as respetivas características ou propriedades de cada objeto, segundo a normalização presente a nível nacional, sendo esta um meio essencial para o estabelecimento de regras comuns e conferindo uniformidade, é fundamental para que o empreendimento apresente maior qualidade e segurança [8].

\section{Enquadramento conceptual}

A classificação surge como uma forma de caracterizar um objeto através de uma relação bem definida, sendo que essa avaliação deve ser feita mediante regras ou seguindo um conjunto de normas estabelecidas. Deste modo, a criação de uma terminologia para determinada área de conhecimento é um ponto fundamental para a consolidação de um domínio técnico. A correta 
definição das designações e os seus respetivos inter-relacionamentos constituem uma referência indispensável para o desenvolvimento do setor [9]. Os sistemas de classificação de informação da construção (CICS) podem ter níveis de ordens de composição, concebendo-se uma estrutura hierarquicamente composta, porém podem também ter níveis de detalhe, divididos em estruturas hierarquicamente organizadas (classes e subclasses). Numa estrutura hierárquica composta, as classes subordinadas constituem subclasses com elementos que fazem parte da classe subordinante, enquanto numa estrutura hierarquicamente organizada, as classes subordinadas formam subclasses do tipo da classe subordinante [1]. Para que um sistema de classificação de informação seja considerado como tal, são necessários determinados requisitos que os caracterizam. Um CICS é sustentado por tabelas incorporando determinadas formas de classificação, referentes a diversas áreas na conceção de um projeto, seguindo a metodologia de classificação do BIM. Qualquer CICS deve conter uma codificação simples, intuitiva e flexível, permitir a aplicação isolada das tabelas ou entre as tabelas, integrar uma base de dados de terminologias, conceitos, descrições e ser orientado por objetos segundo as metodologias BIM. Sendo abrangida a sua complexidade e dimensão nos trabalhos do setor AECO [10]. Apesar de, em Portugal, ainda não existir nem uma regulamentação governamental definida e obrigatória para a utilização do BIM, nem um Sistemas de Classificação de Informação da Construção (CICS) nacional, devido à sua importância e à necessidade de implementação na área da Engenharia Civil, a International Organization for Standardization (ISO), através do Comité Técnico 59 (TC59), tem tido essa preocupação e como principal finalidade a criação e desenvolvimento de normas, com vista à uniformização de toda a informação no setor [4]. A nível nacional tem vindo a ser desenvolvidos estudos e trabalhos sobre os CICS [10-13] mostrando a capacidade e importância do sistema de classificação. Destes estudos destacam-se aqueles que contemplam uma proposta para o CICS nacional com a adoção de caraterísticas semelhantes às de alguns sistemas de classificação de informação nacionais e internacionais existentes [14], onde foram ponderados os principais princípios, atributos e vantagens e do qual surge o desenvolvimento do CICS baseado no sistema internacional Uniclass 2015 com a inclusão das vantagens de outros sistemas e do auxílio da ferramenta portuguesa ProNIC [15]. A ISO 12006-2:2015 orienta a conceção e o desenvolvimento dos sistemas de classificação de informação da construção e permite que estes sejam aplicáveis a empreendimentos e a serviços de engenharia e de ordenamento do território, durante todo o ciclo de vida de um empreendimento (preconceção, conceção, documentação, construção, operação, manutenção e demolição) e dos trabalhos de construção associados [1]. Neste sentido, o CICS nacional proposto deve contemplar os seguintes princípios gerais: i) Estar em conformidade com a norma ISO12006-2:2015; ii) Incluir uma classificação composta por tabelas hierarquicamente organizadas; iii) Abranger a complexidade e dimensão dos trabalhos do setor AECO nacional; iv) Ser dotado de uma codificação simples, intuitiva e flexível para acomodar novas entradas; v) Permitir a aplicação isolada das tabelas ou de tabelas relacionadas entre si; vi) Integrar uma base de dados de terminologias, conceitos e descrições $[10,12,15]$.

\section{Proposta para modelo de classificação nacional}

O CICS proposto baseia-se na análise da ISO 12006-2:2015 [1], na medida em que esta dá as diretrizes para a incorporação de todas as fases do processo construtivo. Esta norma define uma estrutura para sistemas de classificação de informação relacionada com a indústria da 
construção e identifica um conjunto de tabelas recomendadas, bem como as suas nomenclaturas para um universo abrangente de classes de objetos da construção. Além desta referência, o modelo de classificação proposto resulta da revisão de outra proposta de metodologia na fase inicial [10], do estudo dos princípios e organização do sistema de classificação do Reino Unido, Uniclass 2015 [14], e do contributo da ferramenta nacional ProNIC [16]. Na Tabela 1 encontram-se resumidas as tabelas de classificação propostas pela Norma ISO 12006-2:2015 [1], pela Uniclass 2015, bem como as características das 13 tabelas classificativas propostas para um possível CICS, de acordo com a realidade portuguesa.

Tabela 1: Proposta para o CICS nacional e correspondência com a ISO 12006-2:2015 e a Uniclass 2015

\begin{tabular}{|c|c|c|c|c|}
\hline ISO 12006-2:2015 & Uniclass 2015 & $\begin{array}{l}\text { CICS nacional } \\
\text { (proposta) }\end{array}$ & $\begin{array}{l}\text { Princípio de } \\
\text { especialização }\end{array}$ & Definição \\
\hline $\begin{array}{c}\text { Construction } \\
\text { information (A.2) }\end{array}$ & $\begin{array}{l}\text { Form of } \\
\text { information } \\
\text { (FI) }\end{array}$ & $\begin{array}{c}\text { Formas de } \\
\text { Informação (FI) }\end{array}$ & Conteúdo & $\begin{array}{l}\text { Estrutura as formas de } \\
\text { comunicar }\end{array}$ \\
\hline $\begin{array}{c}\text { Construction } \\
\text { products (A.3) }\end{array}$ & Products (Pr) & Produtos (Pr) & Função & $\begin{array}{l}\text { Especifica produtos individuais } \\
\text { utilizados }\end{array}$ \\
\hline $\begin{array}{c}\text { Construction } \\
\text { agents (A4) }\end{array}$ & - & Agentes (Ag) & $\begin{array}{l}\text { Função que } \\
\text { desempenha }\end{array}$ & $\begin{array}{l}\text { Especifica os envolvidos no } \\
\text { processo construtivo }\end{array}$ \\
\hline $\begin{array}{c}\text { Construction aids } \\
\text { (A5) }\end{array}$ & $\begin{array}{c}\text { Tools and } \\
\text { Equipment (TE) }\end{array}$ & $\begin{array}{c}\text { Ferramentas e } \\
\text { equipamentos (FE) }\end{array}$ & Função & $\begin{array}{l}\text { Descreve objetos que são } \\
\text { aplicados }\end{array}$ \\
\hline Management (A.6) & $\begin{array}{l}\text { Project } \\
\text { management } \\
(\mathrm{PM})\end{array}$ & $\begin{array}{l}\text { Gestão e Direção } \\
\text { (GD) }\end{array}$ & $\begin{array}{l}\text { Atividades de } \\
\text { gestão ou } \\
\text { direção }\end{array}$ & $\begin{array}{c}\text { Especifica as atividades de } \\
\text { gestão }\end{array}$ \\
\hline $\begin{array}{l}\text { Construction } \\
\text { process (A.7) }\end{array}$ & - & $\begin{array}{c}\text { Processos de } \\
\text { construção (Po) }\end{array}$ & $\begin{array}{l}\text { Atividades de } \\
\text { construção }\end{array}$ & $\begin{array}{l}\text { Descreve atividades ligadas à } \\
\text { construção }\end{array}$ \\
\hline $\begin{array}{c}\text { Construction } \\
\text { complexes (A.8) }\end{array}$ & Complexes $(\mathrm{Co})$ & $\begin{array}{l}\text { Empreendimentos } \\
(\mathrm{Em})\end{array}$ & Função & $\begin{array}{l}\text { Descreve os projetos de forma } \\
\text { geral }\end{array}$ \\
\hline $\begin{array}{l}\text { Construction } \\
\text { entities (A.9) }\end{array}$ & Entities (En) & Entidades (En) & Função & Descreve coisas concretas \\
\hline Built spaces (A.10) & $\begin{array}{c}\text { Spaces/ } \\
\text { locations }(\mathrm{SL})\end{array}$ & $\begin{array}{l}\text { Espaços/ locais } \\
\text { (EL) }\end{array}$ & Função & $\begin{array}{c}\text { Descreve locais onde podem ser } \\
\text { consideradas atividades }\end{array}$ \\
\hline $\begin{array}{c}\text { Construction } \\
\text { elements (A.11) }\end{array}$ & $\begin{array}{c}\text { Elements/ } \\
\text { functions }(\mathrm{EF})\end{array}$ & $\begin{array}{c}\text { Elementos/ } \\
\text { Funções (E/F) }\end{array}$ & Posição e forma & $\begin{array}{c}\text { Reúne os principais } \\
\text { componentes de uma estrutura }\end{array}$ \\
\hline $\begin{array}{l}\text { Work results } \\
\quad(\mathrm{A} .12)\end{array}$ & - & - & & \\
\hline $\begin{array}{c}\text { Construction } \\
\text { properties }(A .13)\end{array}$ & - & Propriedades $(\mathrm{Pp})$ & $\begin{array}{l}\text { Função que } \\
\text { desempenha }\end{array}$ & $\begin{array}{c}\text { Estrutura as propriedades e } \\
\text { características dos objetos } \\
\text { físicos }\end{array}$ \\
\hline- & Activities (Ac) & - & - & \\
\hline- & Systems (Ss) & Sistemas (Ss) & Função & $\begin{array}{l}\text { Componentes que no seu } \\
\text { conjunto cria elemento ou } \\
\text { desempenha função }\end{array}$ \\
\hline- & $C A D(\mathrm{Zz})$ & $\mathrm{CAD}(\mathrm{Zz})$ & Função & Organiza ficheiros CAD \\
\hline
\end{tabular}


As tabelas encontram-se identificadas pelas suas nomenclaturas, isto é, o par de códigos correspondentes à tabela; a designação da tabela; o princípio de especialização proposto; a sua definição [12], e permitem organizar a informação em diferentes classes com informações diversas, estruturadas de modo idêntico [15]. Apresentam-se nas secções seguintes alguns exemplos de tabelas já propostas para o desenvolvimento de um CICS nacional.

\subsection{Produtos de construção}

As tabelas "Produtos" Pr apresentam os dados primários dos objetos virtuais: a sua constituição e quais as propriedades que podem ser vinculadas a estes. Deve haver uma tentativa de abranger quase todos os materiais usualmente utilizados na construção, ou pelo menos os de maior comercialização. A tabela Pr serve para classificar os produtos ou componentes que irão ser incorporados ou se tenciona incorporar nas instalações. Esta tabela pode ser utilizada para o desenvolvimento de bases de dados de produtos, elaboração de catálogos de produtos de construção, catalogação de Normas de produtos de construção, especificações de produtos de construção, informação genérica de produtos de construção e elaboração de encomendas de produto de construção [12]. A tabela Pr fica organizada segundo a sua função e estruturada de acordo com os produtos que estejam em conformidade com as especificações técnicas harmonizadas e que cumpram as exigências definidas no RPC, isto é, que se encontram abrangidos por uma ENh ou contenham uma ETA, para que o produto possa estar em conformidade e conter marcação CE [17]. Apresenta-se a título de exemplo a tabela Pr genérica proposta, ao nível de grupo. O desenvolvimento completo das tabelas encontra-se patente em [17].

Tabela 2: Proposta genérica para a tabela Pr do CICS Nacional, adaptado de [17]

\begin{tabular}{lc}
\hline Código & Grupo/Descrição \\
\hline Pr_15 & Produtos preparatórios \\
Pr_20 & Produtos Estruturais e Gerais \\
Pr_25 & Produtos para Exterior \\
Pr_30 & Produtos para Entradas \\
Pr_35 & Produtos para Revestimentos e Acabamentos \\
Pr_40 & Produtos paraSinalização, Equipamento Sanitários e Acessórios, Mobiliário e Equipamentos \\
Pr_45 & Produtos paraFlora e Fauna \\
Pr_50 & Produtos Suplementares à Construção \\
Pr_60 & Produtos para Fontes de Serviços \\
Pr_65 & Produtos para Distribuição de Serviços \\
Pr_70 & Produtos para Saída de Serviços \\
Pr_75 & Produtos para Controlo de Serviços \\
Pr_80 & Produtos para Serviços Gerais \\
Pr_85 & Produtos para Engenharia de Processos \\
\hline Pr_90 & Produra Gestão de Instalações Simples \\
\hline
\end{tabular}




\subsection{Propriedades}

A tabela "Propriedades" Pp apresenta os dados primários dos objetos virtuais: a sua constituição e quais as propriedades que podem ser vinculadas a estes. Deve haver uma tentativa de abranger quase todos os materiais usualmente utilizados na construção, ou pelo menos os de maior comercialização. A tabela Pp permite estruturar as propriedades e características dos objetos físicos [10]. Esta pode ser utilizada para organização técnica de documentos, estruturação de base de dados de produtos, estruturação de tabelas adicionais de produtos por atributos primários e definição de exigências para projetos e recursos em geral [12]. Na conceção da tabela Propriedades destaca-se o grupo "30-Propriedades essenciais", com foco no estudo da normalização dos produtos de construção e no que esta avalia, ou seja, as propriedades essenciais dos produtos de construção, e contribui para a informação que a tabela de Propriedades deve conter com informação detalhada da tabela de Produtos do CICS nacional [17]. Apresenta-se a título de exemplo a tabela genérica Pp proposta, ao nível de grupo. $\mathrm{O}$ desenvolvimento completo das tabelas encontra-se patente em [17].

Tabela 3: Proposta genérica para a tabela Pp do CICS Nacional, adaptado de [17]

\begin{tabular}{ccc}
\hline Código & Grupo & Descrição \\
\hline Pp_20 & Propriedades contextuais e ambientais. & $\begin{array}{c}\text { Descreve onde do objeto está colocado em termos } \\
\text { contextuais e ambientais }\end{array}$ \\
Pp_30 & Propriedades essenciais & $\begin{array}{c}\text { Descreve as propriedades funcionais do objeto, } \\
\text { descrição da composição interna e desempenho } \\
\text { Descreve como foi feita a aplicação do objeto na } \\
\text { construção e a sua capacidade na atividade }\end{array}$ \\
Pp_40 & Propriedades das aplicações e das \\
atividade & $\begin{array}{c}\text { Descreve as operaçães e manutenções que o } \\
\text { objeto deve ter }\end{array}$ \\
Pp_50 & Propriedades de operação e & manutenção \\
Pp_55 & Propriedades administrativa & $\begin{array}{c}\text { Descreve o objeto em relação à sua } \\
\text { comercialização }\end{array}$ \\
\hline
\end{tabular}

\subsection{Formas de informação}

Na tabela Formas de Informação (FI) encontram-se itens de legislação, documentos e dados que devem ser gerados pelas empresas envolvidas ao longo do processo de projeto e outra documentação necessária no processo construtivo. Através dela torna-se mais fácil a elaboração de sistemas de tratamento de dados e de fluxos de processos, os quais podem contribuir para a maior produtividade da gestão do empreendimento [12]. Na tabela FI são apresentadas as designações que traduzem os elementos utilizados como meio de suporte, que leva a todos os intervenientes a informação genérica do projeto. Estão presentes documentos relativos ao planeamento, aos recursos usados, à área financeira, à representação gráfica do projeto, entre outros. Neste sentido, através da tabela FI é fornecida a informação geral do ciclo de vida de um determinado empreendimento [4]. Apresenta-se a título de exemplo a tabela genérica FI proposta. O desenvolvimento completo das tabelas encontra-se patente em [4]. 
Tabela 4: Proposta genérica para a tabela FI do CICS Nacional, adaptado de [4]

\begin{tabular}{|c|c|c|}
\hline Código & Grupo & Descrição \\
\hline FI_10 & Comunicação & $\begin{array}{l}\text { Transmissão de mensagens estratégicas entre os colaboradores de } \\
\text { uma determinada empresa, que beneficiem os processos e projetos } \\
\text { a executar, aumentando por sua vez a produtividade e dinamismo } \\
\text { da empresa. }\end{array}$ \\
\hline FI_20 & Condições de contrato & $\begin{array}{l}\text { Conjunturas subjetivas ativas e passivas que devem ser exercidas e } \\
\text { cumpridas de boa-fé e em conformidade com os ditames do } \\
\text { interesse público, nos termos da lei. }\end{array}$ \\
\hline FI_30 & Conjunto de dados & $\begin{array}{l}\text { Conjunto de informação sobre um determinado projeto, por } \\
\text { exemplo, gerido por uma entidade. }\end{array}$ \\
\hline FI_40 & $\begin{array}{l}\text { Informações do } \\
\text { projeto }\end{array}$ & $\begin{array}{l}\text { Informação do conjunto coordenado de documentos escritos e } \\
\text { desenhados, integrando o projeto ordenador e demais projetos, que } \\
\text { definem e caracterizam a conceção funcional, estética e construtiva } \\
\text { de uma obra, bem como a sua inequívoca interpretação por parte } \\
\text { das entidades intervenientes na sua execução. }\end{array}$ \\
\hline FI_50 & Finanças & $\begin{array}{l}\text { Processos, instituições, mercados e instrumentos envolvidos na } \\
\text { transferência de fundos entre pessoas, empresas e governos. }\end{array}$ \\
\hline FI_60 & Informação gráfica & $\begin{array}{l}\text { Imagem visual que pode representar um conjunto de valores de } \\
\text { forma de gráfico facilitando a compreensão das informações. }\end{array}$ \\
\hline FI_70 & $\begin{array}{l}\text { Controlo de } \\
\text { planeamento }\end{array}$ & $\begin{array}{l}\text { Conjunto de procedimentos que garantem o cumprimento do } \\
\text { planeamento estabelecido para um projeto. }\end{array}$ \\
\hline FI_80 & $\begin{array}{l}\text { Planeamento de } \\
\text { projetos }\end{array}$ & $\begin{array}{l}\text { Plano da estrutura e gestão de informações do projeto a executar, } \\
\text { incluindo os processos e procedimentos relacionados. }\end{array}$ \\
\hline FI_90 & $\begin{array}{l}\text { Registo de } \\
\text { informação }\end{array}$ & $\begin{array}{l}\text { Conjunto de dados armazenados relacionados com um determinado } \\
\text { projeto, quer seja em formato digital ou formato físico. }\end{array}$ \\
\hline
\end{tabular}

\subsection{Gestão e Direção de projeto}

A tabela "Gestão e Direção" GD destina-se a classificar as atividades relacionadas com a gestão dos aspetos logísticos, legais e financeiros de todo o processo construtivo, nas diferentes fases deste, tal como projeto, produção, manutenção ou utilização. Através desta tabela pode ser obtida informação sobre comunicação, formação, finanças, contabilidade, controle de custos, análise de custos e planos de produção [12]. Na tabela GD são apresentadas diversas designações direcionadas para os aspetos e atividades de gestão ao longo de todo o procedimento de conceção do empreendimento, sendo a função de cada elemento inserido no projeto, como requisitos de desempenho, relatórios de aprovação dos materiais e serviços, planos de produção e finanças, entre outros exemplos que se destacam. Durante a fase de projeto existe um conjunto de ações que têm de ser obrigatoriamente realizadas, e só assim é possível ter um nível de qualidade e segurança adequado. Todas estas ações têm por trás uma perspetiva teórica ligada diretamente às designações que estão presentes na tabela GD [4]. Apresenta-se a título de exemplo a tabela genérica GD proposta. O desenvolvimento completo das tabelas encontra-se patente em [4]. 
Tabela 5: Proposta genérica para a tabela GD do CICS Nacional, adaptado de [4]

\begin{tabular}{|c|c|c|}
\hline Código & Grupo & Descrição \\
\hline GD_10 & Informações do empreendimento & $\begin{array}{l}\text { Informação desenvolvida durante a fase de projeto e } \\
\text { construção de um empreendimento. }\end{array}$ \\
\hline GD_20 & Pessoal & $\begin{array}{l}\text { Conjunto de elementos que incorporam uma equipa, que } \\
\text { tem a cargo a responsabilidade de conceção, execução, } \\
\text { exploração e manutenção de um projeto. }\end{array}$ \\
\hline GD_30 & $\begin{array}{l}\text { Informação sobre o estaleiro, } \\
\text { terreno e ambiente }\end{array}$ & $\begin{array}{l}\text { Informação dos fatores que condicionam o estado do } \\
\text { estaleiro, terreno e ambiente envolvente do projeto. }\end{array}$ \\
\hline GD_40 & $\begin{array}{l}\text { Informação sobre desempenho, } \\
\text { projeto e aprovações do } \\
\text { empreendimento }\end{array}$ & $\begin{array}{l}\text { Informação sobre aspetos específicos que qualificam e } \\
\text { aprovam um determinado projeto. }\end{array}$ \\
\hline GD_50 & $\begin{array}{l}\text { Informação financeira e } \\
\text { comercial }\end{array}$ & $\begin{array}{l}\text { Informação detalhada, presente em determinados } \\
\text { documentos, sobre as atividades ligadas à logística } \\
\text { financeira, realizadas ao longo do projeto. }\end{array}$ \\
\hline GD_55 & Informação do contrato & $\begin{array}{l}\text { Informação sobre os contratos que são executados num } \\
\text { projeto. }\end{array}$ \\
\hline GD_60 & $\begin{array}{l}\text { Informação de administração de } \\
\text { construção }\end{array}$ & $\begin{array}{l}\text { Informação usada para suportar um ou mais processos } \\
\text { de construção. }\end{array}$ \\
\hline GD_70 & $\begin{array}{l}\text { Teste, pagamentos e informações } \\
\text { de conclusão }\end{array}$ & $\begin{array}{l}\text { Documentos de aprovação de diversos } \\
\text { elementos/serviços inseridos em projeto, bem como a } \\
\text { garantia dos pagamentos efetuados destes mesmos } \\
\text { elementos/serviços. }\end{array}$ \\
\hline GD_80 & Informações de gestão de ativos & $\begin{array}{l}\text { Informação sobre as atividades sistemáticas e } \\
\text { coordenadas, através da qual uma organização realiza a } \\
\text { gestão, de forma otimizada e sustentável, dos seus } \\
\text { ativos e a sua performance associada, riscos e custos, ao } \\
\text { longo do seu ciclo de vida do empreendimento. }\end{array}$ \\
\hline
\end{tabular}

\subsection{Elementos/Funções}

Uma classificação básica é a dos elementos, pois estes constituem a base para estabelecer uma construção virtual e estarão presentes desde o estágio de conceção até ao estágio de utilização. $\mathrm{Ou}$ seja, esta tabela aplica-se para classificar as partes físicas dos edifícios / construções, podendo ser utilizada para análise de soluções técnicas, especificações, informação histórica do projeto e gestão da construção, bem como de custos e mapas de quantidades. A tabela "Elementos/funções" E/F foi complementada com o termo "funções", uma vez que a classificação de objetos de acordo com a tabela elementos assume particular dificuldade, já que determinado objeto pode ser classificado como um elemento e ter mais do que uma função. Ao agregar a noção de "função" passa a ser possível classificar "elementos com a função de", deste modo reduzem-se incongruências e aumenta-se a abrangência e flexibilidade da tabela. Ao introduzir este conceito é possível estabelecer a ligação entre os Elementos / Funções e os produtos, criando a possibilidade de classificar mais objetos [12]. Os grupos da tabela Elementos/Funções estão organizados de acordo o seu fabrico e a sua função, e seguem a 
estrutura das tabelas Elementos/Funções e Sistemas do Uniclass 2015 [10]. Apresenta-se a título de exemplo a tabela genérica $\mathrm{E} / \mathrm{F}$ proposta. $\mathrm{O}$ desenvolvimento completo das tabelas encontra-se patente em [10].

Tabela 6: Proposta genérica para a tabela E/F do CICS Nacional, adaptado de [10]

\begin{tabular}{lc}
\hline Código & Grupo/Descrição \\
\hline E/F_15 & Preparação \\
E/F_20 & Elementos Estruturais \\
E/F_25 & Elementos de confinamento de espaço \\
E/F_30 & Elementos de cobertura, pisos e pavimento \\
E/F_32 & Impermeabilização e acabamentos em gesso \\
E/F_35 & Escadas e rampas \\
E/F_37 & Reservatórios elevados e torre \\
E/F_40 & Sinalização, acessórios, mobília e equipamentos \\
E/F_45 & Elementos de fauna e flora \\
E/F_50 & Recolha e eliminação de resíduos \\
E/F_55 & Abastecimento canalizado \\
E/F_60 & Gquecimento, arrefecimento e refrigeração \\
E/F_65 & Ventilação e ar condicionado \\
E/F_70 & Energia elétrica e iluminação \\
E/F_75 & Comunicações, segurança e proteção \\
E/F_80 & Transportes \\
E/F_85 & Engenharia de processos \\
E/F_90 & Gestão de serviços \\
\hline & \\
\hline &
\end{tabular}

\section{Conclusões}

Em Portugal é cada vez mais notória a ascensão das TIC, que têm sofrido diversas alterações com o desenvolvimento da metodologia BIM. Todas as modificações que tem vindo a acontecer são um passo indispensável para a formação de um CICS nacional, que promova a interoperabilidade e devidamente estruturado sob o ponto de vista técnico. Pode-se afirmar que, caso exista uma base de dados com as referências do projeto nas tabelas normalizadas do CICS, tais como, os produtos de construção utilizados, se torne simples e objetivo alcançar um bom 
planeamento do projeto, de intervenção ou reabilitação, dado que toda a informação passa estar organizada e à disponibilidade dos potenciais intervenientes, obtendo-se um projeto com menores custos, redução do tempo de obra e sendo uma mais-valia para o setor da construção. A existência de um CICS nacional torna-se imprescindível para o desenvolvimento da normalização do setor da construção de modo a que se consiga um planeamento e uma estrutura de todo o seu ciclo de vida, desenvolvendo uma base de dados com referências do projeto evitando erros durante o processo construtivo. Desta forma, promover-se-á a uniformidade entre o setor da construção a nível nacional e entre os vários países. Todas as operações procedentes à criação de um CICS nacional, não acontecem de forma rápida tendo de existir um enquadramento adequado ao dinamismo do setor. Um processo como este pode demorar alguns anos até alcançar a maturidade pretendida. A proposta apresentada no âmbito do presente trabalho, incorporando as tabelas Pp, Pr, FI, GD e E/F, teve como objetivo dar seguimento ao desenvolvimento de um CICS para implementação generalizada no setor AECO nacional. Com a informação adequada e normalizada, utilizada em todas as tabelas propostas, é possível a obtenção de uma codificação uniforme e singular dos objetos e mais eficiente, com um nível de erros e omissões bastante reduzido.

\section{Agradecimentos}

Os autores agradecem reconhecidos os elementos fornecidos pelo Eng. ${ }^{\circ}$ Henrique Nunes, pela Eng. ${ }^{a}$ Teresa Poejo, pela Eng. ${ }^{a}$ Catarina Gomes, pelo Eng. ${ }^{\circ}$ Frederico Mesquita para o desenvolvimento do presente artigo.

\section{Referências}

[1] ISO 12006-2:2015 - Building construction - Organization of information about construction works - Part 2: Framework for classification. International Organization for Standardization.

[2] ISO 12006-3:2007 - Building construction - Organization of information about construction works - Part 3: Framework for object-oriented information. International Organization for Standardization.

[3] Poejo, T; Couto, P.; Falcão Silva, M.J.; Pinho, F. (2018). Contributos para um CICS Nacional, em conformidade com a Norma ISO 12006, Artigo 36, PTBIM 2018, IST, Lisboa, Portugal.

[4] Mesquita, F. (2019). Contributos para o CICS nacional.Tabelas de Formas de Informação e de Gestão e Direção de Projeto, Dissertação de mestrado, FCT-UNL.

[5] ISO 19650-1:2018, Organization and digitalization of information about buildings and civil engineering works, including building information modelling (BIM) - Information management using building information modelling - Part 1: Concepts and principles, CENEuropean Commission for Standardization, Bruxelas, 1st edition.

[6] ISO 19650-2:2018. Organization and digitalization of information about buildings and civil engineering works, including building information modelling (BIM) - Information management using building information modelling - Part 2: Delivery phase of assets, CENEuropean Commission for Standardization, Bruxelas, 1st edition. 
[7] CEN/ TC 442, (2017). Building Information Modeling - Level of Information Need, Part 1: Concepts and principles.

[8] Gomes, A.; Couto, P.; Falcão Silva, M.J.; Pinho, F. (2018). O BIM e a necessidade de um CICS nacional: Tabelas de produtos, Artigo 37, PTBIM 2018, IST, Lisboa, Portugal.

[9] GS1 Brasil, (2009). Classificação Global de Produtos. A linguagem global para classificação de mercadorias. Associação Brasileira de Automação.

[10]Nunes, H. (2016). Sistemas de Classificação de Informação da Construção. Proposta de metodologia orientada para objetos BIM. Departamento de Engenharia Civil, FCT-UNL.

[11] Monteiro, M. (1998). Classificação da informação na indústria da construção - Perspetivas e percursos", Dissertação de mestrado, FEUP, Porto, 1998.

[12]Poêjo, T. (2017). Contributos para um Sistema de Classificação de Informação da Construção Nacional, em conformidade com a Norma ISO 12006, Dissertação de mestrado, Departamento de Engenharia Civil, FCT UNL, Lisboa.

[13]Pereira, R. (2013). Sistemas de classificação na construção. Sintese comparada demétodos", Dissertação de mestrado, Departamento de Engenharia Civil, FEUP, Porto.

[14]Delany, S. (s/d). Classification, NBS BIM Toolkit. [Online]. Disponível em: https://toolkit.thenbs.com/articles/classification/.

[15]Nunes, H.; Couto, P.; Falcão Silva, M.J.; Pinho, P. (2016) Proposta de sistema de classificação nacional orientado para objeto BIM, $1^{\circ}$ Congresso Português de Building Information Modelling, Universidade do Minho, Guimarães, Portugal, pp. 143-152.

[16]Couto, P., (2009). ProNIC®, Sistema de Geração e Gestão de Informação Técnica para Cadernos de Encargos.

[17] Gomes, A. (2018). Contributos para um CICS Nacional: Tabela de produtos de construção e de propriedades. Dissertação de mestrado, FCT UNL, Lisboa.

[18]Gomes, A; Couto, P.; Falcão Silva, M.J.; Pinho, F. (2018). O BIM e a necessidade de um CICS nacional: Tabela de produtos, Artigo 37, PTBIM 2018, IST, Lisboa, Portugal. 